08-094

\title{
Some Neglected Axioms in Fair Division
}

\author{
John W. Pratt
}

Copyright $(92008$ by John W. Pratt.

Working papers are in draft form. This working paper is distributed for purposes of comment and discussion only. It may not be reproduced without permission of the copyright holder. Copies of working papers are available from the author. 


\section{Some neglected axioms in fair division}

John W. Pratt

Harvard Business School

Abstract. Conditions one might impose on fair allocation procedures are introduced. Nondiscrimination requires that agents share an item in proportion to their entitlements if they receive nothing else. The "price" procedures of Pratt (2007), including the Nash bargaining procedure, satisfy this. Other prominent efficient procedures do not. In two-agent problems, reducing the feasible set between the solution and one agent's maximum point increases the utility cost to that agent of providing any given utility gain to the other and is equivalent to decreasing the dispersion of the latter's values for the items he does not receive without changing their total. One-agent monotonicity requires that such a change should not hurt the first agent, limited monotonicity that the solution should not change. For prices, the former implies convexity in the smaller of the two valuations, the latter linearity. In either case, the price is at least their average and hence spiteful.

Keywords: Fair division, efficient allocation, nondiscrimination axiom, monotonicity axioms, envyfree, spite, bargaining solutions.

Acknowledgment. I am grateful to Richard Zeckhauser for helpful comments.

Mailing address: 2 Gray Gardens East, Cambridge, MA 02138

jpratt@hbs.edu 


\section{Introduction and summary}

We consider allocation and bargaining problems and introduce conditions that one might expect fair procedures to satisfy. The first condition, "nondiscrimination," applies to allocation. In the simplest case it asserts that, if two agents with equal entitlements receive probability shares of the same item and no chance of any other, then their shares should be equal. It is a very special case of envy-free and hence is satisfied by what we shall call here "price procedures," which are defined below and by Pratt (2007) and include the Nash bargaining procedure. It is not satisfied by other prominent efficient procedures, however. Symmetric problems with just two items and an even number of agents provide a striking example. Furthermore, envy-free completely determines the solution in such problems, and nearly determines it in many other problems with just two items. It does not do so, however, in problems with just two items and two agents. Indeed, when one agent is indifferent between the two items, these problems afford a natural way to assess a price function. The bounds on a price function implied by a finite number of such assessments are easily derived but disappointingly weak. The convexity implied by a monotonicity condition introduced below strengthens them considerably but still leaves much to smoothing.

The remaining conditions we consider apply to two agents and arbitrary feasible sets. They are weakenings of the Kalai-Smorodinsky (1975) monotonicity axiom, which asserts that, if the feasible set is enlarged without changing either agent's maximum utility, neither agent should lose. Suppose instead that the feasible set is enlarged only on one side of the solution in joint utility space, that is, between the solution and one agent's maximum point. (In allocation problems with additive utilities, this region corresponds to the items allocated to the other agent.) "Limited monotonicity" asserts that neither agent should lose, or equivalently, the allocation should not change. "One-agent monotonicity" asserts that the first agent should not lose. The following example motivates these definitions. Suppose one agent receives two items whose values (utilities) to him are equal and to the other are unequal, say $c$ and $d$. How should the allocation change if $c$ and $d$ are replaced by their average? The replacement reduces the feasible region and increases the utility cost to the first agent of providing any given utility gain to the second, while $c+d$ is the second agent's foregone utility in both cases, and how it is divided between the two items in question does not matter to either agent. One might think, then, that the allocation should not change, or at least the first agent should not lose. These are limited monotonicity and oneagent monotonicity respectively. As this example suggests, enlarging the feasible region over part of the frontier is equivalent to increasing the dispersion of one agent's values for the corresponding items without changing their total. (We assume throughout that an agent's utility for an allocation is the sum of his values for the items he receives.)

For price procedures, limited monotonicity implies that the price of an item is a linear combination of the two agents' valuations, while one-agent monotonicity implies that the price is convex in the smaller valuation when the larger is fixed. In either case, uniqueness implies that the price is at least the average of the two valuations. Hence a price procedure that satisfies even the one-agent monotonicity condition cannot satisfy also the "no spite" condition that an agent should not be penalized for receiving an item no one else wants. For equal entitlements and a differentiable frontier, limited monotonicity and no-spite together imply that at any efficient solution, the slope of the frontier equals 
minus the ratio of the two agents' utilities foregone, or equivalently, on the tangent to the frontier at the solution, the solution is midway between the point where one agent receives his maximum and the point where the other does. This alone sometimes determines the solution. Similar results hold for any entitlements and frontiers.

Section 2 introduces nondiscrimination. Results for two items appear in Section 3, for two agents in Section 4. The Appendix defines the procedures mentioned here and quotes the results of Pratt (2007) used, with one correction. The idea of the price procedures, roughly, is to scale the agents' utility functions so that the market clears with the agents' purchases proportional to their entitlements when the price of each item is a specified increasing function of its two highest scaled values $x$ and $y$ of the form $f(x, y)=x g(y / x)$ where $0 \leq y \leq f(x, y) \leq x$. For such a procedure to be unique, it is necessary that $g(w) / \sqrt{ } w$ be weakly decreasing in $w$ and sufficient that it be strictly decreasing. Borderline cases are special and not needed in this paper.

\section{Nondiscrimination}

For any allocation problem, fairness would seem to require at least the following condition.

Nondiscrimination axiom: If two agents receive shares of the same item and no other, their shares should be proportional to their entitlements.

Envy-free is clearly much stronger. Price procedures are envy-free and hence nondiscriminatory, but other notable procedures are not even nondiscriminatory, as the following theorem states.

Theorem 1. Price procedures, including the Nash procedure, satisfy the nondiscrimination axiom. The equipartite (Adjusted Winner, equal-proportion-of-potential), discrete Raiffa, and KMP procedures, do not.

A particularly striking example of discrimination occurs in symmetric situations with equal entitlements. Thus, in Table 1, item 1 is shared between the two agents who prefer it under all the procedures shown, as symmetry demands, but the one who prefers it more gets the smaller share under the equipartite, discrete Raiffa, and KMP procedures. Though Agent 1 prefers item 1 by a factor of 10, he receives a significantly smaller share of it than Agent 2, who prefers it by a factor of only 2. This is not just unfair, it is positively perverse.

Table 1. Discriminatory procedures

\begin{tabular}{|c|c|c|c|c|c|c|c|c|}
\hline \multirow[b]{4}{*}{ Agent 1} & \multirow{2}{*}{\multicolumn{2}{|c|}{ values }} & \multicolumn{6}{|c|}{ shares } \\
\hline & & & \multirow{2}{*}{\multicolumn{2}{|c|}{$\begin{array}{c}\text { equipartite } \\
\text { item } 1 \text { item } 2\end{array}$}} & \multirow{2}{*}{\multicolumn{2}{|c|}{$\begin{array}{l}\text { discrete Raiffa } \\
\text { item } 1 \text { item } 2\end{array}$}} & \multirow{2}{*}{\multicolumn{2}{|c|}{$\begin{array}{c}\text { KMP } \\
\text { item } 1 \text { item } 2\end{array}$}} \\
\hline & ite & item 2 & & & & & & \\
\hline & 10 & 1 & $11 / 26$ & 0 & 0.45 & 0 & 0.40 & 0 \\
\hline Agent 2 & 2 & 1 & $15 / 26$ & 0 & 0.55 & 0 & 0.60 & 0 \\
\hline Agent 3 & 1 & 2 & 0 & $15 / 26$ & 0 & 0.55 & 0 & 0.60 \\
\hline Agent 4 & 1 & 10 & 0 & $11 / 26$ & 0 & 0.45 & 0 & 0.40 \\
\hline
\end{tabular}




\section{Sharing two items fairly among any number of individuals}

Assume now that $n$ agents are to share two items probabilistically, the agents' utilities are additive, and all agents desire both items. Generalizing Table 1, we see that in symmetric problems with equal entitlements, two items, and an even number of agents none of whom are indifferent between the two, all efficient nondiscriminatory procedures agree and share each item equally among the agents who prefer it. As background to further analysis, we observe that:

Theorem 2. An allocation of two items is efficient if and only if all agents whose valuations for item 1 relative to item 2 exceed some critical value receive shares of item 1 only, while all agents whose relative valuations fall below this critical value receive shares of item 2 only. Agents at the critical value, if any, may receive shares of both items.

Now suppose the agents have equal entitlements. If an efficient allocation is nondiscriminatory, then all agents on the same side of the critical value must receive equal shares. If an efficient allocation is envy-free, then not only is it nondiscriminatory, but also which agents share item 1 only is determined, and similarly for item 2. Furthermore, the shares in the two items of the agents at the critical value are limited to a non-convex quadrilateral region determined by four inequalities expressing the requirements that they should neither envy nor be envied by the agents just below and just above the critical value. Price procedures are envy-free in all additive allocation problems and hence they agree here except at the critical value, where they may differ. The equipartite, discrete Raiffa, and KMP procedures are discriminatory in general, as noted above, and hence not envy free. The case of only two types of agents is a trivial exception, and the only one at least for the equipartite procedure.

Pratt (2007, Example 4.1) suggests a way of assessing a price function based on assessing what would be fair in situations with two items and two agents, one of whom is indifferent between the two items. To obtain the whole price function in this way without further assumptions would, however, require an assessment for every degree of preference by the other agent. Let $g(w)$ be the price of an item worth 1 to one agent and $w<1$ to another. What can one do with a finite number of assessed values, say $g\left(w_{i}\right)$ for $0<w_{1}<w_{2}<\ldots<w_{m}<1$ ? The uniqueness condition implies that $g\left(w_{i}\right) / \sqrt{ } w_{i}$ must be decreasing in $i$, and of course $g\left(w_{i}\right)$ must be increasing in $i$. Given such values $g\left(w_{i}\right)$, upper and lower bounds on the whole price function are easily developed from the conditions that $g(w) / \sqrt{ }_{w}$ be decreasing and $g(w)$ increasing in $w$. Specifically, for all $i, g\left(w_{i}\right)\left(w / w_{i}\right)^{1 / 2}$ is a lower bound on $g(w)$ for $w<w_{i}$ and an upper bound for $w>w_{i}$, and these bounds are tight where they lie between $g\left(w_{i-1}\right)$ and $g\left(w_{i+1}\right)$. Elsewhere in the interval $\left(w_{i-1}, w_{i}\right)$ the lower bound is $g\left(w_{i-1}\right)$ and the upper bound is $g\left(w_{i}\right)$. These bounds can be approached arbitrarily closely, but their graphs have horizontal steps. If $g$ is convex with $g^{\prime}(1) \leq 1 / 2$, which is stronger than $g(w) / \sqrt{ } w$ decreasing, then the piecewise linear bounds obtained in the usual way are tighter. In either case, a suitable smoothness condition would greatly narrow them. Finding and justifying such a condition is an open problem. 


\section{Monotonicity conditions for two agents}

We start with an example. Suppose two agents are to share three items, which they value as in Table 2. Assume $0 \leq c \leq d \leq 1$. The efficient frontier is generated by giving A either a share of item 1 , or item 1 and a share of item 2, or items 1 and 2 and a share (possibly 0 ) of item 3. Since items 1 and 2 together are worth no more to either agent than item 3, fairness dictates restricting consideration to the last case. Suppose, then, that A receives items 1 and 2 while the two agents share item 3. Since B's utility for items 1 and 2, the items denied him, is $c+d$, he doesn't care how this total is divided between them. A cares still less. It might be argued therefore that their shares of item 3 should depend only on $c$ $+d$. We call this condition limited monotonicity.

Table 2. Two agents share three items

$\frac{\text { values }}{\text { item } 1 \text { item } 2 \text { item } 3}$

Agent A

Agent B

prices

$\frac{\text { shares }}{\text { item } 1 \text { item } 2 \text { item } 3}$

$1 \quad 1 \quad \alpha$

$0 \quad 0 \quad 1-\alpha$ expenditures

$2 \alpha+g(c)+g(d)$

$2(1-\alpha)$

For a price procedure with price function $g$, the market clears with equal weights on $\mathrm{A}$ and $\mathrm{B}$ and $\alpha=1 / 2-[g(c)+g(d)] / 4$.

Then limited monotonicity requires that $\alpha$ depend only on $c+d$. This is equivalent to requiring that $g$ be linear. It follows that the price of an item with scaled values $u$ and $v$ is $f(u, v)=p u+(1-p) v$ with $1 / 2 \leq p$ $\leq 1$, the constraints on $p$ being necessary and sufficient for uniqueness (by Corollary A2 in the Appendix). Thus all price procedures that satisfy the condition are spiteful, the least spiteful being the simple average of the two highest values, which still charges an agent at least half his value for an item no matter how little other agents value it, indeed, even if they consider it worthless.

Notice that the frontier is piecewise linear with vertices where A's utility is $0,1,2$, or 4 . The slope of the first (left) piece is $-c$, of the second is $-d$, and of the third is -1 . As $c$ and $d$ change with $c+$ $d$ fixed, the vertex $\mathrm{V}_{1}$ where A's utility is 1 goes up and down but the other vertices are fixed. If, for example, $c$ decreases and $d$ increases by an equal amount, then $\mathrm{V}_{1}$ rises and the feasible region expands. Specifically, the frontier corresponding to items allocated to A moves up, reducing or leaving unchanged the utility cost to A of providing any given utility gain to B. It might be argued that B should not lose by such a change, and hence, in the example, $\alpha$ should not increase. We call this condition one-agent monotonicity. It is weaker than limited monotonicity. In the example above, it is equivalent to the condition that the price function $g$ be convex, and we shall prove that the price must again be at least the average of the two highest values. (We remark in passing that when $c=d$, Table 2 becomes equivalent to the situation in the last paragraph of Section 3.)

These conditions generalize immediately to arbitrary two-agent "bargaining" problems, that is, arbitrary convex, closed (finite) feasible sets in two dimensions. One-agent monotonicity requires that if the feasible set is enlarged (frontier increased) between the solution and one agent's maximum point (without changing the maximum), that agent should not lose. Limited monotonicity requires that neither agent should lose by such an enlargement, or equivalently, the solution should not change. Full (Kalai-Smorodinsky) monotonicity requires that, if the feasible set is enlarged in any way without changing either agent's maximum utility, neither agent should lose. Clearly the first is weaker than the second and the second than the third. 
For price procedures we have:

Theorem 3. In two-agent additive allocation problems, a price procedure satisfies one-agent monotonicity if and only if the price function is convex, and limited monotonicity if and only if the price function is linear. In either case, the price is at least the average of the two highest scaled valuations, and this price function satisfies both conditions.

Proof. The only ifs are proved in the course of the discussion above. The ifs are proved in the Appendix. The last sentence of the theorem can be proved by means of Theorems 2 and 3 of Pratt (2007), quoted in the Appendix, and the fact that if $g$ is a convex (or linear) function on $[0,1]$ such that $g(w) / \sqrt{ } w$ is decreasing and $g(1)=1$, then $g(w) \geq(1+w) / 2$.

As the example at the beginning of this section suggests, monotonicity is related to dispersion. Note first that, for two agents, every bargaining set can be approximated arbitrarily closely by the bargaining set of an additive allocation problem where one agent has equal values for all items, as follows. Assume for convenience and without loss of generality that A's utility is scaled so that its range over the feasible set is $[0,1]$. Let there be $m$ items, each of value $1 / m$ to A. Let $v(u)$ be B's utility at the efficient point where A's utility is $u$ and let B's value for item $i$ be $b_{i}=v((i-1) / m)-v(i / m)$. Then $b_{i} \leq b_{i+1}$ by concavity of $v$, and $\Sigma_{j \leq i} b_{j}=v(0)-v(i / m)$. Thus the frontier of the additive allocation problem with these values agrees with $v$ at $i / m$ and is piecewise linear between them. Defining "increased dispersion" by second-order dominance, we have:

Theorem 4. In a two-agent additive allocation problem, increasing the efficient frontier over an interval is equivalent to increasing the dispersion of one agent's values in that interval without changing their mean.

Proof. Since $v(0)-v(i / m)$ is the sum of the $b_{j}$ to the left of $i / m$, the frontier is a piecewise linear approximation to the integrated quantile function of the $b_{i}$, inverted and displaced vertically by $v(0)$. Increasing the frontier over an interval is therefore equivalent to decreasing the integrated quantile function in an interval. By an argument like that in Machina and Pratt (1997, Section 3), this is equivalent to increasing the integrated cumulative function over the same interval, and hence to increasing the dispersion in that interval without changing the mean.

In the context of additive allocation problems, one-agent monotonicity requires that increasing the dispersion of one agent's values for items he does not receive should not hurt that agent; limited monotonicity requires that the solution should not change. It is assumed throughout that the total of the first agent's values does not change and that their ratios to the second agent's values remain smaller for the items he does not receive than for the items he receives.

What follows from limited monotonicity and no spite alone?

Theorem 5. In a two-agent allocation or bargaining problem, if an efficient solution satisfies the limited monotonicity and no-spite conditions, then the slope of the frontier at the solution equals minus the ratio of the two agents' utilities foregone times the ratio of their entitlements. Specifically, if the agents have utilities $u$ and $v$ and the frontier is given by $v(u)$ and the solution is $\left(u_{0}, v_{0}\right)$, then $v^{\prime}\left(u_{0}\right)=-\rho\left(v_{\max }-\right.$ $\left.v_{0}\right) /\left(u_{\max }-u_{0}\right)$ where $u_{\max }$ and $v_{\max }$ are the maximum feasible values of $u$ and $v$ and $\rho$ is the ratio of the entitlement of the agent with utility $v$ to that of the agent with utility $u$. If $v^{\prime}\left(u_{0}\right)$ does not exist, then it is replaced by the interval between the lower and upper derivatives and $=$ by $\ni$ (includes). 
Proof. By limited monotonicity, the solution does not change when the feasible region is enlarged as far as the tangent to the frontier at the solution. No spite then implies that the solution is the same as that for the triangular region bounded by the tangent and the lines $u=u_{1}$ and $v=v_{1}$ where $\left(u_{1}, v_{\max }\right)$ and $\left(u_{\max }, v_{1}\right)$ are the intersections of the tangent with the lines $v=v_{\max }$ and $u=u_{\max }$. This is equivalent to a problem where the agents share a single item worth $u_{\max }-u_{1}$ to one and $v_{\max }-v_{1}$ to the other. In this problem, their shares must be proportional to their entitlements. Therefore the solution is $\rho$ times as far from $\left(u_{1}, v_{\max }\right)$ as from $\left(u_{\max }, v_{1}\right)$. In particular, $\left(v_{0}-v_{1}\right)=\rho\left(v_{\max }-v_{0}\right)$. Since $\left(v_{0}-v_{1}\right) /\left(u_{0}-u_{\max }\right)=$ $v^{\prime}\left(u_{0}\right)$ or lies between the lower and upper derivatives of $v(u)$ at $u_{0}$, the conclusion follows.

To obtain a feeling for the implications of Theorem 5, consider a feasible region whose frontier is given by the hyperbola $\left(u_{\max }-u\right)\left(v_{\max }-v\right)=1$ between two points $\mathrm{L}$ and $\mathrm{R}$, say, with $\mathrm{L}$ to the left of $\mathrm{R}$. To the left of $\mathrm{L}$, let the frontier be the tangent to this hyperbola at $\mathrm{L}$, and similarly to the right of $\mathrm{R}$. Then every point on the frontier between $\mathrm{L}$ and $\mathrm{R}$ satisfies the condition of Theorem 5 , since $v^{\prime}=-\left(v_{\max }\right.$ $-v) /\left(u_{\max }-u\right)$. If the frontier between $\mathrm{L}$ and $\mathrm{R}$ is replaced by an inscribed polygon, then the vertices and one point on each side of the polygon satisfy the condition. On the other hand, if $\left(u_{\max }-u\right)\left(v_{\max }-\right.$ $v$ ) is unimodal on the frontier, then the condition is satisfied only at its maximum.

\section{Conclusion}

Pitfalls lurk in the field of fair division. Not all conditions one might hope for can be satisfied simultaneously. However, some apparently plausible and widely proposed axioms and procedures have consequences whose undesirability clearly goes far beyond what can be excused in this way.

\section{Appendix}

To avoid irrelevant complications, we assume unless stated otherwise that we are dealing with allocation problems where all agents have non-negative values for all items, additive utility functions, and equal entitlements, though some statements apply to more general feasible sets in the space of joint utilities. We first define the procedures discussed here and then quote some results we need for price procedures, including one correction. Pratt (2007) gives more details, proofs, and references.

The Nash procedure maximizes the product of the agents' utility gains. It is equivalent to a price procedure with price equal to maximum scaled value. The equipartite procedure chooses the efficient point that gives each agent the same proportion of his maximum possible utility gain; if no such point exists, we treat the procedure as undefined. The discrete Raiffa procedure moves first to the point where each of the $n$ agents gains a fraction $1 / n$ of his maximum possible gain, recalculates the maximum possible gains at the point reached, and repeats until the frontier is reached or approached. We believe the results above would apply to smaller or continuous moves, but have not investigated them. The KMP (Kohlberg-Maschler-Perles) procedure moves in the (unique) direction where the rates at which each agent gains utility and loses possible further gain have the same ratio for all agents, stopping when the efficient frontier is reached.

A price procedure with price function $g$ picks a scaling of each agent's utility function, and sharing probabilities if needed, in such a way that the agents receive allocations of total value proportional to their entitlements when: (1) the value (price) of an item is $f(x, y)=x g(y / x)$ where $x$ and $y$ are its two highest scaled values and $y \leq x$; (2) each item is allocated to the agent or shared among the agents with the highest scaled value for it. Equivalently, for two agents and a general bargaining set, 
suppose B's maximum feasible utility is $v(u)$ when A's utility is $u$. The price procedure allocates $u_{\lambda}$ to A and $v\left(u_{\lambda}\right)$ to B after finding a scaling $\lambda$ of $v$ and a value $u_{\lambda}$ such that

$$
\lambda v^{\prime}\left(u_{\lambda}\right)=-1 \text { and } \int_{\leq} g\left(-\lambda v^{\prime}\right)=\int_{\geq} \lambda g\left(-1 /\left(\lambda v^{\prime}\right)\right)
$$

where the first (second) integral is over the range below (above) $u_{\lambda}$.

Pratt (2007) gives the following results, numbered as here except for the letter A. Unfortunately Corollary 2 as stated there is wrong as regards the linear case. For convenience, we restate the entire corollary with correction, and replace $f(1, w)$ by $g(w)$. "Unique" means up to a common scale factor.

Theorem A2. If $g(w) / \sqrt{ } w$ is strictly decreasing, then the scaling and the agents' expected utilities are unique.

Theorem A3. If $g(w) / \sqrt{ } w$ is not weakly decreasing, then there exist problems with as few as two agents and three items where the scaling and the agents' expected utilities are not unique.

Corollary A2. With $q=1-p$, the scaling and the agents' expected utilities are unique for $f(u, v)=u^{p} v^{q}$ if $1 / 2<p \leq 1$; for $f(u, v)=p u+q v$ if $1 / 2 \leq p \leq 1$; for $f(u, v)=u^{p}(v+\varepsilon u)^{q} /(1+\varepsilon)^{q}$ with $\varepsilon>0$, if $(1-\varepsilon) / 2 \leq p$ $\leq 1$; for $f(u, v)=u^{p} v^{q} /(1-\varepsilon+\varepsilon v / u)^{q}$ with $0<\varepsilon<1$, if $p>1 / 2$. They are not always unique for these functions if the parameter values are outside the ranges given.

Details for the proof of Theorem 3. To prove the ifs, consider the approximation described earlier with $m$ items of equal value to A. Note that increasing the frontier over an interval of A's utility is equivalent to increasing the dispersion of B's values for the items corresponding to that interval (Theorem 4). If the initial solution allocates these items to $\mathrm{A}$, the total of their prices at the initial scaling will not decrease if the price function is convex and will be unchanged if the price function is linear. If the total of the prices is unchanged, the solution will be unchanged. If the total of the prices increases, then $\mathrm{A}$ is paying more than $\mathrm{B}$, in which case one expects the solution to change so that $\mathrm{A}$ receives less and $\mathrm{B}$ receives more. We now prove this.

Assume $g(w) / \sqrt{ } w$ is decreasing in $w$, so that the solution is unique (Theorem A2). We will show further that, for smaller $\lambda$, the first integral in (2) is less than the second. Suppose without loss of generality that equality holds for $\lambda=1$. Then for $\lambda<1$, the first equation in (2) implies $u_{\lambda}>1$, since $v^{\prime}$ is decreasing, and the monotonicity condition implies $g(\lambda u) \geq \sqrt{ } \lambda g(u)$. Therefore

$$
\int_{\leq} g\left(-\lambda v^{\prime}\right)>\int_{\leq 1} g\left(-\lambda v^{\prime}\right) \geq \sqrt{ } \lambda \int_{\leq 1} g\left(-v^{\prime}\right)=\sqrt{ } \lambda \int_{\geq 1} g\left(-1 / v^{\prime}\right) \geq \int_{\geq 1} \lambda g\left(-1 /\left(\lambda v^{\prime}\right)\right)>\int_{\geq} \lambda g\left(-1 /\left(\lambda v^{\prime}\right)\right)
$$

Now suppose that $v$ is increased in the range $\left[0, u_{\lambda}\right]$. This is a mean-preserving increase in risk. If also $g$ is convex, then the first integral of (2) and (3) is increased. It follows by the previous paragraph that the solution $\lambda$ cannot decrease. Therefore $u_{\lambda}$ cannot increase, i.e., B cannot lose.

\section{References}

Kalai, Ehud and Meir Smorodinsky (1975). "Other Solutions to Nash's Bargaining Problem," Econometrica 43, 513-518.

Machina, Mark J. and John W. Pratt (1997). “Increasing Risk: Some Direct Constructions," Journal of Risk and Uncertainty 14, 103-127.

Pratt, John W. (2007). "Fair (and Not So Fair) Division,” Journal of Risk and Uncertainty 35, 203-236. 